b) There are very limited teaching facilities in astronomy in view of the acute scarcity of qualified astronomy teachers and also of equipment, which is quite expensive. A more liberal attitude for supplementing is needed.

c) Presently, astronomy seems to have limited job potential in India. The postgraduates in astronomy have no avenues for entering the teaching profession, as neither the subject nor the post of astronomy teacher exists in most of the colleges and universities. Unless they are created, there is little hope for availability of qualified staff in astronomy.

d) Astronomy books are not easily available. They should be made available at low cost.

e) Usually astronomy is mistaken for astrology. Ignorance of the subject and its importance among the general public and those who matter could be removed by educating them through the media.

\title{
TEACHING ELEMENTARY ASTRONOMY IN VILLAGE PRIMARY SCHOOLS IN INDIA
}

Dr. M.F. Ingham, Institute of Astronomy, University of Cambridge, U.K., reported on the work of Professor V.G. Kulkarni and his colleagues at the Homi Bhabha Centre for Science Education, none of whom could attend this meeting. They are preparing some excellent material for teaching elementary astronomy in village primary schools in India. The text of Dr. Ingham's paper was not provided to us, but we have included, below, some of the discussion which followed this interesting paper. Further information can be obtained from Professor V.G. Kulkarni, Homi Bhabha Centre for Science Education, Tata Institute for Fundamental Research, Homi Bhabha Road, Bombay 400 005, India - Editors.

\section{Discussion}

J.V. Narlikar: The Homi Bhabha Centre for Science Education gets financial support from the Sir Dorab Tata (charitable) Trust. It also gets considerable infrastructure support from the Department of Atomic Energy of the Government of India, which supports the Tata Institute for Fundamental Research.

J.L. Dunlap: Did the materials suggest the use of common objects to model the concepts presented to the children?

M.F. Ingham: The drawings, for example, for day/night, show the use of a ball and a lamp. This showed a method by which the ideas could be confirmed.

C. Iwaniszewska: How would the language (local language) problem be dealt with 
when producing the cheap booklets for villagers in India?

M.F. Ingham: Although the captions to the pictures were written in English, the language of the text was very simple and could easily be translated into any other language or dialect.

\section{TEACHING AND POPULARIZING ASTRONOMY IN EGYPT AND OTHER ARAB COUNTRIES}

\section{A. Aiad}

Astronomy Department, Faculty of Science, Cairo University, Egypt

The recent astronomy of the Arab countries began by the last decade of the 19th century in Alger and the first decade of the 20th century in Helwan. Two Arab countries have been members of the IAU, namely Egypt (since 1925) and Iraq (since 1976). Saudi Arabia, Algeria, and Morocco became members in 1988. We restrict ourselves here to the teaching and popularizing of astronomy.

\section{Egypt}

In Egypt there is a single department of astronomy; since 1937 it has belonged to the Faculty of Science of Cairo University. A B.Sc. in astronomy requires two years' study of mathematics and physics followed by two more years devoted mainly to astronomy, including a small project. Elementary courses for other sciences, such as geography and geophysics, are also taught at Cairo University and the American University in Cairo.

Alazhar (Islamic) University has approved an astronomy department. The first staff candidate has recently been sent in a mission to University College London for his Ph.D.

Daily astronomical shows and specials shows for schools have been presented in the planetarium of Cairo since 1967.

The Astronomical Society of Egypt was founded in 1976 and has published a quarterly Arabic magazine, "World of Astronomy and Space," since 1983. A one or two-week school for 30-60 physics and mathematics teachers has been held annually by the society since 1983 . The teachers attend lectures, receive practical training, and visit the planetarium and observatory. They have been more interested in lectures illustrated with slides, video, and movies than with normal lectures. They were most impressed when they saw the heavenly bodies and stellar spectra through the telescope.

After each school the teachers become interested in astronomy, apply to be members of the society, and, of course, popularize astronomy more among their 